PROCEEDINGS OF THE

AMERICAN MATHEMATICAL SOCIETY

Volume 135, Number 3, March 2007, Pages 743-753

S 0002-9939(06)08535-2

Article electronically published on August 28, 2006

\title{
LINEARIZED STABILITY OF TRAVELING CELL SOLUTIONS ARISING FROM A MOVING BOUNDARY PROBLEM
}

\author{
Y. S. CHOI AND ROGER LUI
}

(Communicated by M. Gregory Forest)

\begin{abstract}
In 2003, Mogilner and Verzi proposed a one-dimensional model on the crawling movement of a nematode sperm cell. Under certain conditions, the model can be reduced to a moving boundary problem for a single equation involving the length density of the bundled filaments inside the cell. It follows from the results of Choi, Lee and Lui (2004) that this simpler model possesses traveling cell solutions. In this paper, we show that the spectrum of the linear operator, obtained from linearizing the evolution equation about the traveling cell solution, consists only of eigenvalues and there exists $\mu>0$ such that if $\lambda$ is a real eigenvalue, then $\lambda \leq-\mu$. We also provide strong numerical evidence that this operator has no complex eigenvalue.
\end{abstract}

\section{INTRODUCTION}

In this paper we study the eigenvalue problem

$$
\left\{\begin{aligned}
\mathcal{L} \phi-p(x) c+a(x) \phi^{\prime}(0)+b(x) \phi^{\prime}(1) & =\lambda \phi, \\
-\alpha c+\beta\left(\phi^{\prime}(0)-\phi^{\prime}(1)\right) & =\lambda c,
\end{aligned}\right.
$$

with $\phi(0)=\phi(1)=0$ for some particular differential operator $\mathcal{L}$, known functions $a, b, p$, and known constants $\alpha$ and $\beta$. The precise form of the equations is given in (2.7). Here, $(\phi, c)$ is an "eigenfunction" consisting of a function $\phi(x)$ and a constant $c$ and $\lambda$ is the eigenvalue. The above system arises naturally as one performs linearized stability analysis on the traveling cell solution of a moving boundary problem modeling cell motility. The function $\phi$ and the constant $c$ correspond to perturbations of the traveling cell solution and the cell size, respectively. The cell motility model was developed by Mogilner and Verzi in [3] and the traveling cell solution of the model was shown to exist by Choi, Lee, and Lui in [1. We refer readers to these papers for details of the model and an existence proof of the traveling cell solutions.

Received by the editors September 3, 2004 and, in revised form, September 26, 2005.

2000 Mathematics Subject Classification. Primary 35P15; Secondary 35R35, 37L15.

Key words and phrases. Cell motility, moving boundary problem, traveling cell, linearized operator, eigenvalues.

The first author's research was partially supported by NIH grant no. 5P41-RR013186-07.

The second author's research was partially supported by NSF grant no. DMS-0456570.

(C)2006 American Mathematical Society Reverts to public domain 28 years from publication 
Under some simplifying conditions, it can be shown (see 2] that the MogilnerVerzi model reduces to the following moving boundary problem:

$$
\left\{\begin{aligned}
b_{t} & =\rho K\left(b b_{x} / \xi_{0}\right)_{x}-\gamma_{b} b, \\
b(r, t) & =b_{0}, \quad b(f, t)=b_{0} \\
r^{\prime} & =V_{d}-\rho K b_{x}(0, t) / \xi_{0} \\
f^{\prime} & =\frac{L}{\ell}-\rho K b_{x}(\ell, t) / \xi_{0} .
\end{aligned}\right.
$$

This system of equations is assumed to hold in the domain $\{(x, t): r(t)<x<$ $f(t), t>0\}$, where $r(t), f(t)$ denote the rear and front end of the cell, respectively. Also, $b$ denotes the length density of the bundled filaments inside the cell, $\rho, K, \xi_{0}, \gamma_{b}, b_{0}, V_{d}$ and $L$ are positive constants each with their own biological meaning, and $\ell(t)=f(t)-r(t)$ is the length of the cell. Global existence of this moving boundary problem has been proved by Choi, Groulx and Lui in [2].

In [1], Choi, Lee, and Lui proved the existence of traveling cell solutions for the Mogilner-Verzi model, which implies the existence of traveling cell solutions for (1.2). Traveling cell solutions are special solutions of the form $b(x, t)=\bar{b}(x-k t)$. Biologically, this means that the cell maintains a constant shape and moves with a constant velocity $k$. If the traveling cell has constant length $\bar{\ell}$ and moves with velocity $\bar{k}$, then $r(t)=\bar{k} t$ and $f(t)=\bar{\ell}+k t$. Substituting this form of solution into (1.2) and using $y=x-\bar{k} t$, the traveling cell system is

$$
\left\{\begin{aligned}
0 & =\rho K\left[\bar{b} \bar{b}^{\prime} / \xi_{0}\right]^{\prime}+\bar{k} \bar{b}^{\prime}-\gamma_{b} \bar{b}, \\
\bar{b}(0) & =b_{0}, \quad \bar{b}(\bar{\ell})=b_{0}, \\
\bar{k} & =V_{d}-\rho K \bar{b}_{x}(0) / \xi_{0}, \\
\bar{k} & =\frac{L}{\ell}-\rho K \bar{b}_{x}(\bar{\ell}) / \xi_{0},
\end{aligned}\right.
$$

where $^{\prime}=d / d y$. For simplicity, we also assume that $L=\rho K / \xi_{0}=1$ in (1.2) and (1.3) so that we do not have to carry these constants around in our future calculations.

\section{THE LINEARIZED SYSTEM}

To study the stability of the traveling cell solution, we linearize (1.2) about the traveling cell solution. To this end, we straighten out the moving boundaries by 
letting $\bar{x}=(x-r(t)) /(f(t)-r(t))$ and then write $x$ for $\bar{x}$. System (1.2) becomes

$$
\left\{\begin{aligned}
b_{t} & =\frac{1}{\ell^{2}}\left(\frac{b^{2}}{2}\right)_{x x}+\left(\frac{r^{\prime}+x \ell^{\prime}}{\ell}\right) b_{x}-\gamma_{b} b \\
b(0, t) & =b_{0}, \quad b(1, t)=b_{0} \\
r^{\prime} & =V_{d}-\frac{b_{x}(0, t)}{\ell} \\
f^{\prime} & =\frac{1}{\ell}-\frac{b_{x}(1, t)}{\ell}
\end{aligned}\right.
$$

Let $\tilde{b}(x)=\bar{b}(x \bar{\ell})$. From (1.3), we obtain a system of equations for $\tilde{b}$, which we shall not display. Let $u=b-\tilde{b}, \zeta_{1}=\ell-\bar{\ell}, \zeta_{2}=r-\bar{k} t$. After some tedious calculations, the linearized system is

$$
\left\{\begin{array}{l}
u_{t}=\mathcal{L} u+\left[-\frac{2}{\bar{\ell}^{3}}\left(\frac{\tilde{b}^{2}}{2}\right)_{x x}-\frac{\bar{k}}{\bar{\ell}^{2}} \tilde{b}_{x}\right] \zeta_{1}+\frac{\tilde{b}_{x}}{\bar{\ell}}\left[x \zeta_{1 t}+\zeta_{2 t}\right] \\
\zeta_{1 t}=-\frac{V_{d}}{\bar{\ell}} \zeta_{1}-\left(\frac{u_{x}(1, t)-u_{x}(0, t)}{\bar{\ell}}\right) \\
\zeta_{2 t}=-\frac{u_{x}(0, t)}{\bar{\ell}}+\frac{\tilde{b}_{x}(0)}{\bar{\ell}^{2}} \zeta_{1}
\end{array}\right.
$$

where

$$
\mathcal{L} u=\frac{1}{\bar{\ell}^{2}}(\tilde{b} u)_{x x}+\frac{\bar{k}}{\bar{\ell}} u_{x}-\gamma_{b} u,
$$

and $u(0, t)=u(1, t)=0$ for $t \geq 0$. Substituting $\zeta_{1 t}, \zeta_{2 t}$ from (2.2 $\left.\mathrm{b}\right)$, (2.2 $)$ into $(2.2$ ) and employing the equation for $\tilde{b}$ derived from (1.3 $)$, the linearized system may be written as

$$
\left\{\begin{aligned}
u_{t} & =\mathcal{L} u-p(x) \zeta_{1}-E_{1} u, \\
\zeta_{1 t} & =-\frac{V_{d}}{\bar{\ell}} \zeta_{1}+E_{2} u,
\end{aligned}\right.
$$

where $u(0, t)=u(1, t)=0, p(x)=2 \gamma_{b} \tilde{b} / \bar{\ell}-(1-x) V_{d} \tilde{b}_{x} / \bar{\ell}^{2}$,

$$
\begin{gathered}
E_{1} u=\frac{\tilde{b}_{x}}{\bar{\ell}^{2}}\left[x u_{x}(1, t)+(1-x) u_{x}(0, t)\right] \quad \text { and } \\
E_{2} u=\frac{u_{x}(0, t)-u_{x}(1, t)}{\bar{\ell}} .
\end{gathered}
$$

Let $u(x, t)=\phi(x) e^{\lambda t}$ and $\zeta_{1}(t)=c e^{\lambda t}$. Then (2.4) becomes

$$
\left\{\begin{aligned}
\mathcal{L} \phi-p(x) c-E_{1} \phi & =\lambda \phi, \\
-\frac{V_{d}}{\bar{\ell}} c+E_{2} \phi & =\lambda c,
\end{aligned}\right.
$$

where $\phi(0)=\phi(1)=0$. The main results of this paper are the following propositions. 
Proposition 2.1. The spectrum of the operator on the left of (2.7) consists only of eigenvalues.

Proposition 2.2. Let $\lambda$ be an eigenvalue of (2.7). (a) Suppose $c=0$. Then $\mathcal{R} e(\lambda) \leq-\gamma_{b}$. (b) Suppose $c \neq 0$ and $\lambda$ is real. Then $\lambda \leq \max \left\{-\gamma_{b},-V_{d} / \bar{\ell}\right\}$.

Remark. We conjecture that system (2.7) consists only of real eigenvalues and in the last section of this paper, we provide strong numerical evidence to support our conjecture. From Proposition 2.2, all real eigenvalues correspond to stable modes and therefore, if our conjecture is true, the traveling wave solution is linearly stable. However, because (2.7) is nonlocal, meaning that $E_{1}, E_{2}$ involve $\phi$ at the boundary points, it is not clear if this implies local stability of the traveling cell solution.

Proof of Proposition 2.1. Let the linear operator on the left-hand side of (2.7) be denoted by $\mathcal{A}$. It is clear that $\mathcal{A}$ has only a discrete spectrum if and only if $\mathcal{A}-$ $a I$ does for a large positive constant $a$. We now show that the inverse operator $(\mathcal{A}-a I)^{-1}$ is defined and compact.

Consider the equation $\mathcal{A} u-a u=f$, where $f=\left(f_{1}, f_{2}\right) \in C[0,1] \times \mathbf{R}$ is given and we need to solve for $u=(\phi, c)$. Solving for $c$ from (2.7b) and substituting the result into $(2.7 \mathrm{~b})$, we obtain

$$
\mathcal{L} \phi-a \phi-p(x) \frac{\bar{\ell}}{V_{d}+a \bar{\ell}}\left(E_{2} \phi-f_{2}\right)-E_{1} \phi=f_{1} .
$$

Simplifying, this equation can be written in the form

$$
\phi^{\prime \prime}-a \phi=\beta\left\{p_{1}(x, a) \phi^{\prime}(0)+p_{2}(x, a) \phi^{\prime}(1)+p_{3}(x) \phi+p_{4}(x) \phi^{\prime}+f_{3}(x, a)\right\}
$$

with boundary conditions $\phi(0)=\phi(1)=0$. Here, $\beta=1, p_{i}(\cdot, a), i=1, \ldots, 4$, and $f_{3}(\cdot, a)$ are known functions belonging to $C[0,1]$ and their $C[0,1]$ bounds are independent of $a$ for $a \geq 0$.

We now establish a priori bounds on the solution $\phi$ for $0 \leq \beta \leq 1$. Letting $\beta g$ denote the right side of (2.8), we have $\phi(x)=\beta \int_{0}^{1} G(x, y, a) g(y) d y$, where the Green's function $G(x, y, a)$ can be explicitly calculated. It follows that $\phi^{\prime}(x)=$ $\beta \int_{0}^{1} G_{x}(x, y, a) g(y) d y$ even though $G_{x}$ has a discontinuity when $x=y$. Using the explicit form of $G$, we have

$$
\left|\phi^{\prime}(x)\right| \leq \beta\|g\|_{C[0,1]} \int_{0}^{1}\left|G_{x}(x, y, a)\right| d y \leq \frac{M \beta}{\sqrt{a}}\|g\|_{C[0,1]},
$$

where $M>0$ is independent of $a \geq 0$ and $\beta$. From the definition of $g$, we have

$$
\left\|\phi^{\prime}\right\|_{C[0,1]} \leq \frac{M_{1} \beta}{\sqrt{a}}\left(\|\phi\|_{C[0,1]}+\left\|\phi^{\prime}\right\|_{C[0,1]}+\|f\|_{C[0,1]}\right) \leq \frac{M_{2} \beta}{\sqrt{a}}\left(\left\|\phi^{\prime}\right\|_{C[0,1]}+\|f\|_{C[0,1]}\right)
$$

since $\phi(0)=0$. Let $a=4 M_{2}^{2}$. Since $0 \leq \beta \leq 1$, we obtain the a priori estimate

$$
\left\|\phi^{\prime}\right\|_{C[0,1]} \leq \beta\|f\|_{C[0,1]} .
$$

This immediately leads to a bound on $\|\phi\|_{C[0,1]}$. Using (2.8), we obtain an a priori bound on the $C^{2}[0,1]$ norm of $\phi$. This bound is independent of $\beta$. Since (2.8) has a solution when $\beta=0$ with a non-zero degree, using a homotopy argument in standard degree theory, we obtain the existence of a solution to (2.8). The estimate (2.10) implies uniqueness of such a solution. Hence, the inverse operator $(\mathcal{A}-a I)^{-1}: C[0,1] \times \mathbf{R} \rightarrow C[0,1] \times \mathbf{R}$ is well defined and the bound (2.10) implies that it is a compact operator. The proof of the proposition is complete. 
We close this section by deriving some properties of the coefficients in (2.7).

Lemma 2.1. (i) $\tilde{b}$ is strictly convex on $[0,1]$ and there exists $x^{*} \in(0,1)$ such that $\tilde{b}$ is strictly decreasing on $\left(0, x^{*}\right)$ and strictly increasing on $\left(x^{*}, 1\right)$.

(ii) $p$ defined above satisfies $p(x) \geq 0$.

Proof. (i) Let $v=\tilde{b}_{x}$. Then $v$ satisfies the equation $\tilde{b} v_{x x}+3 v v_{x}+\bar{k} \bar{\ell} v_{x}-\gamma_{b} \bar{\ell}^{2} v=0$. From Lemma 3.1 of [1], $V_{d}<\bar{k}<V_{p}(\bar{\ell})$ so that $(1.3 \mathrm{k}$, d) imply that $v(0)<0$ and $v(1)>0$. From the differential equation above, $v$ cannot have a positive maximum or a negative minimum, hence $v$ is increasing on $[0,1]$ and $\tilde{b}$ is convex. From Hopf's boundary point lemma, it is easy to see that $\tilde{v}_{x}>0$ and $\tilde{b}$ is strictly convex on $[0,1]$. The rest of (i) is obvious.

(ii) The fact that $p \geq 0$ is clear from its definition on the interval $\left[0, x^{*}\right]$. On the interval $\left[x^{*}, 1\right]$, we have

$$
\begin{aligned}
\bar{\ell}^{2} p(x) & =\frac{2}{\bar{\ell}}\left(\frac{\tilde{b}^{2}}{2}\right)_{x x}+2 \bar{k} \tilde{b}_{x}-(1-x) V_{d} \tilde{b}_{x} \\
& =\frac{2}{\bar{\ell}} \tilde{b} \tilde{b}_{x x}+\frac{2}{\bar{\ell}} \tilde{b}_{x}^{2}+\left[2 \bar{k}-(1-x) V_{d}\right] \tilde{b}_{x} \geq 0
\end{aligned}
$$

since $\bar{k}>V_{d}$ and $\tilde{b}$ is convex. The proof of the lemma is complete.

\section{Proof of Proposition 2.2}

We separate the proof into two cases, when $c=0$ and when $c \neq 0$.

Lemma 3.1. Suppose $c=0$ and $\lambda=\lambda_{1}+i \lambda_{2}$ is an eigenvalue of (2.7). Then $\gamma_{b}+\lambda_{1} \leq 0$.

Proof. From (2.7b) and (2.6), we have $\phi_{x}(0)=\phi_{x}(1)$. From the definition of $E_{1}$, (2.7) may be reduced to a single equation

$$
\frac{1}{\bar{\ell}^{2}}(\tilde{b} \phi)_{x x}+\frac{\bar{k}}{\bar{\ell}} \phi_{x}-\gamma_{b} \phi-\frac{\tilde{b}_{x}}{\bar{\ell}^{2}} \phi_{x}(0)=\lambda \phi
$$

with $\phi(0)=\phi(1)=0$. From (1.3), $\tilde{b}$ is analytic and hence $\phi$ is also analytic and has at most a finite number of zeros on $[0,1]$.

Case 1: $\lambda=0$. Without loss of generality, we may assume that $\phi$ is real. Integrating (3.1), we have $\int_{0}^{1} \phi=0$ and hence $\phi$ must change sign in $[0,1]$. Let $\beta_{0}<1$ be the last interior zero of $\phi$ on $[0,1]$. Without loss of generality, we may assume that $\phi<0$ on $\left(\beta_{0}, 1\right)$. Integrating (3.1) over $\left[\beta_{0}, 1\right]$, we have

$$
\frac{1}{\bar{\ell}^{2}}\left[b_{0} \phi_{x}(1)-\tilde{b}\left(\beta_{0}\right) \phi_{x}\left(\beta_{0}\right)\right]-\gamma_{b} \int_{\beta_{0}}^{1} \phi=\frac{\phi_{x}(0)}{\bar{\ell}^{2}}\left[b_{0}-\tilde{b}\left(\beta_{0}\right)\right] .
$$

Rearranging, we have

$$
\gamma_{b} \int_{\beta_{0}}^{1} \phi=\frac{\tilde{b}\left(\beta_{0}\right)}{\bar{\ell}^{2}}\left[\phi_{x}(0)-\phi_{x}\left(\beta_{0}\right)\right]
$$

The right side is non-negative because $\phi_{x}(0)=\phi_{x}(1) \geq 0$ and $\phi_{x}\left(\beta_{0}\right) \leq 0$ while the left side is negative since $\phi<0$ on $\left(\beta_{0}, 1\right)$. This contradiction implies that 0 is not an eigenvalue if $c=0$. 
Case 2: $\lambda \neq 0$. Assume $\gamma_{b}+\lambda_{1}>0$ and let $\psi(x)=\int_{0}^{x} \phi(t) d t$. Integrating (3.1), we have

$$
\frac{1}{\bar{\ell}^{2}}\left(\tilde{b} \psi_{x}\right)_{x}+\frac{\bar{k}}{\bar{\ell}} \psi_{x}-\gamma_{b} \psi-\frac{\tilde{b}}{\bar{\ell}^{2}} \psi_{x x}(0)=\lambda \psi
$$

with $\psi(0)=\psi(1)=\psi_{x}(0)=\psi_{x}(1)=0$. Without loss of generality we assume that $\psi_{x x}(0)=1$ and $\lambda_{2} \geq 0$. Equation (3.2) has a particular solution $\psi_{p}=-\tilde{b} / \lambda \bar{\ell}^{2}$. Let $\psi=\psi_{p}+\psi_{c}$, where $\psi_{c}$ satisfies

$$
\frac{1}{\bar{\ell}^{2}}\left(\tilde{b} \psi_{c}^{\prime}\right)^{\prime}+\frac{\bar{k}}{\bar{\ell}} \psi_{c}^{\prime}-\gamma_{b} \psi_{c}=\lambda \psi_{c}
$$

and the boundary conditions $\psi_{c}(0)=-\psi_{p}(0), \psi_{c}^{\prime}(0)=-\psi_{p}^{\prime}(0), \psi_{c}(1)=-\psi_{p}(1)$, $\psi_{c}^{\prime}(1)=-\psi_{p}^{\prime}(1)$. Multiplying (3.3) by $w(x)=\exp \left(\int_{0}^{x} \overline{k \ell} / \tilde{b}\right)$, we can convert the equation into the self-adjoint form

$$
\frac{1}{\bar{\ell}^{2}}\left(w \tilde{b} \psi_{c}^{\prime}\right)^{\prime}-\gamma_{b} w \psi_{c}=\lambda w \psi_{c}
$$

Multiplying (3.4) by $\bar{\psi}_{c}$, integrating and then taking the imaginary parts, we obtain

$$
\lambda_{2} \int_{0}^{1} w\left|\psi_{c}\right|^{2}=\left.\frac{1}{\bar{\ell}^{2}}\left\{w \tilde{b} \mathcal{I} m\left(\bar{\psi}_{c} \psi_{c}^{\prime}\right)\right\}\right|_{0} ^{1}=\left.\frac{1}{\bar{\ell}^{2}}\left\{w \tilde{b} \mathcal{I} m\left(\bar{\psi}_{p} \psi_{p}^{\prime}\right)\right\}\right|_{0} ^{1}=0 .
$$

Thus, either $\psi_{c} \equiv 0$ or $\lambda_{2}=0$. In the former case, we have $\psi=\psi_{p}$, which does not satisfy $\psi(0)=0$. Therefore, $\lambda_{2}=0$ and both $\lambda=\lambda_{1}$ and $\psi$ are real. From (3.2), we have

$$
\frac{1}{\bar{\ell}^{2}}\left(\tilde{b} \psi_{x}\right)_{x}+\frac{\bar{k}}{\bar{\ell}} \psi_{x}-\left(\gamma_{b}+\lambda_{1}\right) \psi=\frac{\tilde{b}}{\bar{\ell}^{2}}>0, \quad \psi(0)=0, \quad \psi(1)=0 .
$$

Since $\gamma_{b}+\lambda_{1}>0$, the maximum principle implies that $\psi \leq 0$ on [0,1]. From Hopf's boundary point lemma, either $\psi^{\prime}(0)<0$ and $\psi^{\prime}(1)>0$ or $\psi \equiv 0$. Both cases are impossible because we already know that $\psi^{\prime}(0)=\psi^{\prime}(1)=0$ and $\psi \equiv 0$ won't satisfy (3.6). Thus, $\gamma_{b}+\lambda_{1} \leq 0$ and the proof of the lemma is complete.

Lemma 3.2. If $c \neq 0$ and $\lambda$ is real, then $\lambda \bar{\ell}+V_{d}>0$ and $\lambda+\gamma_{b}>0$ cannot both hold.

Proof. We assume that $\lambda \bar{\ell}+V_{d}>0$ and $\lambda+\gamma_{b}>0$ and arrive at a contradiction. In what follows, we shall call $\alpha$ an interior zero of $\phi$ if $0<\alpha<1, \phi(\alpha)=0$, and $\phi$ changes sign across $\alpha$.

Solving for $c$ from $(2.7 \mathrm{~b})$ and substituting the result into (2.7 $\mathrm{a})$, we have

$$
\frac{1}{\bar{\ell}^{2}}(\tilde{b} \phi)_{x x}+\frac{\bar{k}}{\bar{\ell}} \phi_{x}-\gamma_{b} \phi+\left(\frac{-2 \gamma_{b} \tilde{b}+\lambda x \tilde{b}_{x}}{\lambda \bar{\ell}+V_{d}}\right) E_{2} \phi-\frac{\frac{V_{d} \tilde{b}_{x} \phi_{x}(1)}{\bar{\ell}}+\lambda \tilde{b}_{x} \phi_{x}(0)}{\lambda \bar{\ell}^{2}+V_{d} \bar{\ell}}=\lambda \phi \text {. }
$$

Since $\tilde{b}(0)=\tilde{b}(1)=b_{0}$ and $\int_{0}^{1} x \tilde{b}_{x} d x=b_{0}-\int_{0}^{1} \tilde{b}$, we integrate (3.7) and obtain

$$
E_{2} \phi=\frac{-\left(\lambda+\gamma_{b}\right)\left(\lambda \bar{\ell}^{2}+V_{d} \bar{\ell}\right) \int_{0}^{1} \phi}{b_{0} V_{d}+\left(\lambda+2 \gamma_{b}\right) \bar{\ell} \int_{0}^{1} \tilde{b}}=-\omega\left(\lambda+\gamma_{b}\right) \int_{0}^{1} \phi
$$


where

$$
\omega=\frac{\left(\lambda \bar{\ell}^{2}+V_{d} \bar{\ell}\right)}{b_{0} V_{d}+\left(\lambda+2 \gamma_{b}\right) \bar{\ell} \int_{0}^{1} \tilde{b}}>0
$$

because of our hypotheses. Substituting (3.8) into (3.7) and simplifying, we obtain $(3.10)$

$\frac{1}{\bar{\ell}^{2}}(\tilde{b} \phi)_{x x}+\frac{\bar{k}}{\bar{\ell}} \phi_{x}-\gamma_{b} \phi+\frac{\left(2 \gamma_{b} \tilde{b}-\lambda x \tilde{b}_{x}\right)\left(\lambda+\gamma_{b}\right) \bar{\ell} \int_{0}^{1} \phi}{b_{0} V_{d}+\left(\lambda+2 \gamma_{b}\right) \bar{\ell} \int_{0}^{1} \tilde{b}}-\frac{\frac{V_{d} \tilde{b}_{x} \phi_{x}(1)}{\bar{\ell}}+\lambda \tilde{b}_{x} \phi_{x}(0)}{\lambda \bar{\ell}^{2}+V_{d} \bar{\ell}}=\lambda \phi$.

Equation (3.10) is in the form of $\left(\tilde{b} \phi / \bar{\ell}^{2}\right)_{x x}+\bar{k} \phi_{x} / \bar{\ell}-\left(\gamma_{b}+\lambda\right) \phi+c_{1} \tilde{b}+c_{2} \tilde{b}_{x}+c_{3} x \tilde{b}_{x}=$ 0 . Since $\tilde{b}$ is analytic, so is $\phi$, which therefore has only finite number of zeros on the interval $[0,1]$.

Let $0 \leq \alpha<\beta \leq 1$ be two zeros of $\phi$. Integrating (3.10) over $[\alpha, \beta]$, we have

$$
\begin{gathered}
\frac{1}{\bar{\ell}^{2}}\left[\tilde{b}(\beta) \phi_{x}(\beta)-\tilde{b}(\alpha) \phi_{x}(\alpha)\right]-\left(\gamma_{b}+\lambda\right) \int_{\alpha}^{\beta} \phi+\eta_{\alpha \beta}\left(\gamma_{b}+\lambda\right) \int_{0}^{1} \phi \\
-\frac{\left[V_{d} \phi_{x}(1)+\lambda \bar{\ell} \phi_{x}(0)\right][\tilde{b}(\beta)-\tilde{b}(\alpha)]}{\lambda \bar{\ell}^{3}+V_{d} \bar{\ell}^{2}}=0
\end{gathered}
$$

where

$$
\eta_{\alpha, \beta}=\frac{(\lambda+2 \gamma) \bar{\ell} \int_{\alpha}^{\beta} \tilde{b}-\lambda \bar{\ell}(\beta \tilde{b}(\beta)-\alpha \tilde{b}(\alpha))}{b_{0} V_{d}+(\lambda+2 \gamma) \bar{\ell} \int_{0}^{1} \tilde{b}} .
$$

Let $\alpha_{0}$ and $\beta_{0}$ be the first and last interior zeros of $\phi$ on $[0,1]$, respectively. We may assume that $\phi<0$ on $\left(\beta_{0}, 1\right)$ so that $\phi_{x}(1) \geq 0$ and $\phi_{x}\left(\beta_{0}\right) \leq 0$. Letting $\alpha=\beta_{0}$ and $\beta=1$ in (3.11), we have

$$
\begin{gathered}
\frac{\phi_{x}(1)\left[b_{0} \lambda \bar{\ell}+V_{d} \tilde{b}\left(\beta_{0}\right)\right]}{\left(\lambda \bar{\ell}+V_{d}\right) \bar{\ell}^{2}}-\frac{\tilde{b}\left(\beta_{0}\right) \phi_{x}\left(\beta_{0}\right)}{\bar{\ell}^{2}}-\frac{\lambda \phi_{x}(0)\left[b_{0}-\tilde{b}\left(\beta_{0}\right)\right]}{\lambda \bar{\ell}^{2}+V_{d} \bar{\ell}} \\
-\left(\gamma_{b}+\lambda\right) \int_{\beta_{0}}^{1} \phi+\eta_{1}\left(\gamma_{b}+\lambda\right) \int_{0}^{1} \phi=0 .
\end{gathered}
$$

Letting $\alpha=0$ and $\beta=\alpha_{0}$ in (3.11), we have

$$
\begin{gathered}
\frac{-\phi_{x}(0)\left[b_{0} V_{d}+\lambda \tilde{b}\left(\alpha_{0}\right) \bar{\ell}\right]}{\left(\lambda \bar{\ell}+V_{d}\right) \bar{\ell}^{2}}+\frac{\tilde{b}\left(\alpha_{0}\right) \phi_{x}\left(\alpha_{0}\right)}{\bar{\ell}^{2}}-\frac{V_{d} \phi_{x}(1)\left[\tilde{b}\left(\alpha_{0}\right)-b_{0}\right]}{\lambda \bar{\ell}^{3}+V_{d} \bar{\ell}^{2}} \\
-\left(\gamma_{b}+\lambda\right) \int_{0}^{\alpha_{0}} \phi+\eta_{0}\left(\gamma_{b}+\lambda\right) \int_{0}^{1} \phi=0 .
\end{gathered}
$$

In the above equations $\eta_{0}=\eta_{0 \alpha_{0}}$ and $\eta_{1}=\eta_{\beta_{0} 1}$. We now divide the proof into two cases:

Case 1: $\phi$ has an even number of interior zeros. We may assume that $\phi<0$ on $\left(0, \alpha_{0}\right)$.

Subcase (a): $\alpha_{0}<x^{*}$. We first claim that $b_{0} V_{d}+\lambda \tilde{b}\left(\alpha_{0}\right) \bar{\ell} \geq 0$. This is clear if $\lambda \geq 0$. If $\lambda<0$, then $b_{0} V_{d}+\lambda \tilde{b}\left(\alpha_{0}\right) \bar{\ell} \geq b_{0}\left(V_{d}+\lambda \bar{\ell}\right) \geq 0$ from our hypothesis. Since 
$\phi_{x}(0) \leq 0, \phi_{x}\left(\alpha_{0}\right) \geq 0$ and $\phi_{x}(1) \geq 0$, (3.14) implies that $\eta_{0} \int_{0}^{1} \phi<0$. We claim that

$$
\eta_{0}=\frac{\left(\lambda+2 \gamma_{b}\right) \bar{\ell} \int_{0}^{\alpha_{0}} \tilde{b}-\lambda \bar{\ell} \alpha_{0} \tilde{b}\left(\alpha_{0}\right)}{b_{0} V_{d}+\left(\lambda+2 \gamma_{b}\right) \bar{\ell} \int_{0}^{1} \tilde{b}}>0 .
$$

This is clear if $\lambda \leq 0$. If $\lambda>0$, then since $\tilde{b}$ is decreasing on $\left(0, x^{*}\right), \int_{0}^{\alpha_{0}} \tilde{b}-\alpha_{0} \tilde{b}\left(\alpha_{0}\right)>$ 0 and hence $\eta_{0}>0$. From above, $\int_{0}^{1} \phi<0$. On the other hand, since $\phi_{x}(0) \leq 0$ and $\phi_{x}(1) \geq 0$, (2.6) implies that $E_{2} \phi \leq 0$. From (3.8), $\int_{0}^{1} \phi \geq 0$, which is a contradiction.

Subcase (b): $\alpha_{0}>x^{*}$. Letting $\alpha=\alpha_{0}$ and $\beta=\beta_{0}$ in (3.11) and rearranging, we have

$$
\begin{gathered}
\frac{1}{\bar{\ell}^{2}}\left[\tilde{b}\left(\beta_{0}\right) \phi_{x}\left(\beta_{0}\right)-\tilde{b}\left(\alpha_{0}\right) \phi_{x}\left(\alpha_{0}\right)\right]-\frac{V_{d} \phi_{x}(1)\left[\tilde{b}\left(\beta_{0}\right)-\tilde{b}\left(\alpha_{0}\right)\right]}{\lambda \bar{\ell}^{3}+V_{d} \bar{\ell}^{2}} \\
-\left(\gamma_{b}+\lambda\right) \int_{\alpha_{0}}^{\beta_{0}} \phi+\eta\left(\gamma_{b}+\lambda\right) \int_{0}^{1} \phi-\frac{\lambda \phi_{x}(0)\left[\tilde{b}\left(\beta_{0}\right)-\tilde{b}\left(\alpha_{0}\right)\right]}{\lambda \bar{\ell}^{2}+V_{d} \bar{\ell}}=0,
\end{gathered}
$$

where $\eta=\eta_{\alpha_{0} \beta_{0}}$. First we consider the 2 nd and the 5 th terms on the left-hand side of (3.15). From (3.8), $\phi_{x}(0)=\phi_{x}(1)-\left(\lambda+\gamma_{b}\right) \omega \bar{\ell} \int_{0}^{1} \phi$, we have

$$
\begin{gathered}
-\frac{V_{d} \phi_{x}(1)\left[\tilde{b}\left(\beta_{0}\right)-\tilde{b}\left(\alpha_{0}\right)\right]}{\lambda \bar{\ell}^{3}+V_{d} \bar{\ell}^{2}}-\frac{\lambda \phi_{x}(0)\left[\tilde{b}\left(\beta_{0}\right)-\tilde{b}\left(\alpha_{0}\right)\right]}{\lambda \bar{\ell}^{2}+V_{d} \bar{\ell}} \\
=-\frac{\phi_{x}(1)\left(V_{d}+\lambda \bar{\ell}\right)\left[\tilde{b}\left(\beta_{0}\right)-\tilde{b}\left(\alpha_{0}\right)\right]}{\lambda \bar{\ell}^{3}+V_{d} \bar{\ell}^{2}}+\frac{\lambda\left(\lambda+\gamma_{b}\right) \omega \int_{0}^{1} \phi\left[\tilde{b}\left(\beta_{0}\right)-\tilde{b}\left(\alpha_{0}\right)\right]}{\lambda \bar{\ell}+V_{d}} .
\end{gathered}
$$

From our hypotheses, the first term on the right of the above equation is nonpositive. Since $\phi_{x}\left(\beta_{0}\right) \leq 0$ and $\phi_{x}\left(\alpha_{0}\right) \geq 0$, the first term on the left of (3.15) is also non-positive. Finally, since $\phi<0$ on $\left(0, \alpha_{0}\right)$ and $\left(\beta_{0}, 1\right)$, we have $\int_{\alpha_{0}}^{\beta_{0}} \phi>\int_{0}^{1} \phi$. These facts and (3.15) together imply that

$$
\left(-1+\eta+\frac{\lambda \omega\left[\tilde{b}\left(\beta_{0}\right)-\tilde{b}\left(\alpha_{0}\right)\right]}{\lambda \bar{\ell}+V_{d}}\right) \int_{0}^{1} \phi>0 .
$$

Since $\phi_{x}(0) \leq 0$ and $\phi_{x}(1) \geq 0$, (2.6) implies that $E_{2} \phi \leq 0$. From (3.8), $\int_{0}^{1} \phi \geq 0$ so that (3.17) is still valid without the integral term. Substituting the definitions of $\eta$ and $\omega$ (see (3.12), (3.9)) into (3.17) and simplifying, we have

$$
\begin{aligned}
&-b_{0} V_{d}-\left(\lambda+2 \gamma_{b}\right) \bar{\ell} \int_{0}^{1} \tilde{b}+\left(\lambda+2 \gamma_{b}\right) \bar{\ell} \int_{\alpha_{0}}^{\beta_{0}} \tilde{b}-\lambda \bar{\ell}\left(\beta_{0} \tilde{b}\left(\beta_{0}\right)-\alpha_{0} \tilde{b}\left(\alpha_{0}\right)\right) \\
&+\lambda \bar{\ell}\left[\tilde{b}\left(\beta_{0}\right)-\tilde{b}\left(\alpha_{0}\right)\right]>0
\end{aligned}
$$

which can be rewritten as

$$
-b_{0} V_{d}-2 \gamma_{b} \bar{\ell}\left[\int_{0}^{1} \tilde{b}-\int_{\alpha_{0}}^{\beta_{0}} \tilde{b}\right]-\lambda \bar{\ell}\left[\int_{0}^{1} \tilde{b}-\int_{\alpha_{0}}^{\beta_{0}}(1-x) \tilde{b}_{x}\right]>0 .
$$

If $\lambda \leq 0$, then since $b_{0} V_{d}+\lambda \bar{\ell} \int_{0}^{1} \tilde{b} \geq b_{0}\left(V_{d}+\lambda \bar{\ell}\right)>0$ and $\tilde{b}_{x} \geq 0$ on $\left[x^{*}, 1\right]$, the above inequality produces a contradiction. If $\lambda>0$, the term inside the last 
square bracket in the above inequality is greater than $\int_{0}^{1} \tilde{b}-\int_{x^{*}}^{1}(1-x) \tilde{b}_{x}=\int_{0}^{1} \tilde{b}+$ $\left(1-x^{*}\right) \tilde{b}\left(x^{*}\right)-\int_{x^{*}}^{1} \tilde{b}>0$ so that the above inequality produces a contradiction. This completes the proof of case 1 .

Case 2: $\phi$ has an odd number of interior zeros. We may assume that $\phi>0$ on $\left(0, \alpha_{0}\right)$.

Subcase (a): $E_{2} \phi \leq 0$. Since $\phi_{x}\left(\beta_{0}\right) \leq 0$ and $\int_{\beta_{0}}^{1} \phi<0$, we have, from (3.13),

$$
\frac{\phi_{x}(1) b_{0} \lambda \bar{\ell}}{\bar{\ell}^{2}\left(\lambda \bar{\ell}+V_{d}\right)}+\frac{V_{d} \phi_{x}(1) \tilde{b}\left(\beta_{0}\right)}{\lambda \bar{\ell}^{3}+V_{d} \bar{\ell}^{2}}-\frac{\lambda \phi_{x}(0)\left[b_{0}-\tilde{b}\left(\beta_{0}\right)\right]}{\lambda \bar{\ell}^{2}+V_{d} \bar{\ell}}+\eta_{1}\left(\gamma_{b}+\lambda\right) \int_{0}^{1} \phi<0 .
$$

Replacing $\phi_{x}(1)$ by $\phi_{x}(0)-\bar{\ell} E_{2} \phi$ in the above inequality, simplifying, and using the facts that $\phi_{x}(0) \geq 0, V_{d}+\lambda \bar{\ell} \geq 0$, and (3.8), we have

$$
\begin{aligned}
0 & >\frac{b_{0} \lambda \bar{\ell}+V_{d} \tilde{b}\left(\beta_{0}\right)}{\bar{\ell}^{2}\left(\lambda \bar{\ell}+V_{d}\right)}\left[\phi_{x}(0)-\bar{\ell} E_{2} \phi\right]-\frac{\lambda \phi_{x}(0)\left[b_{0}-\tilde{b}\left(\beta_{0}\right)\right]}{\lambda \bar{\ell}^{2}+V_{d} \bar{\ell}}+\eta_{1}\left(\gamma_{b}+\lambda\right) \int_{0}^{1} \phi \\
& =\frac{\left(V_{d}+\lambda \bar{\ell}\right)}{\bar{\ell}^{2}\left(\lambda \bar{\ell}+V_{d}\right)} \tilde{b}\left(\beta_{0}\right) \phi_{x}(0)-\frac{b_{0} \lambda \bar{\ell}+V_{d} \tilde{b}\left(\beta_{0}\right)}{\bar{\ell}\left(\lambda \bar{\ell}+V_{d}\right)} E_{2} \phi+\eta_{1}\left(\gamma_{b}+\lambda\right) \int_{0}^{1} \phi \\
& \geq \frac{b_{0} \lambda \bar{\ell}+V_{d} \tilde{b}\left(\beta_{0}\right)}{\bar{\ell}\left(\lambda \bar{\ell}+V_{d}\right)} \omega\left(\lambda+\gamma_{b}\right) \int_{0}^{1} \phi+\eta_{1}\left(\gamma_{b}+\lambda\right) \int_{0}^{1} \phi .
\end{aligned}
$$

Substituting in the definitions of $\omega$ and $\eta_{1}$ and rearranging, we have

$$
0>\left\{\frac{\left(V_{d}+\lambda \bar{\ell} \beta_{0}\right) \tilde{b}\left(\beta_{0}\right)+\left(\lambda+2 \gamma_{b}\right) \bar{\ell} \int_{\beta_{0}}^{1} \tilde{b}}{b_{0} V_{d}+\left(\lambda+2 \gamma_{b}\right) \bar{\ell} \int_{0}^{1} \tilde{b}}\right\}\left(\gamma_{b}+\lambda\right) \int_{0}^{1} \phi .
$$

Since the term inside the parenthesis is non-negative, we have $\int_{0}^{1} \phi<0$, which from (3.8) implies that $E_{2} \phi>0$. This contradicts the subcase we are in.

Subcase (b): $E_{2} \phi>0$. Since $\phi_{x}\left(\alpha_{0}\right) \leq 0$ and $\int_{0}^{\alpha_{0}} \phi>0$, we have, from (3.14),

$$
0<\frac{-b_{0} \phi_{x}(0) V_{d}}{\bar{\ell}\left(\lambda \bar{\ell}^{2}+V_{d} \bar{\ell}\right)}-\frac{\lambda \tilde{b}\left(\alpha_{0}\right) \phi_{x}(0)}{\lambda \bar{\ell}^{2}+V_{d} \bar{\ell}}-\frac{V_{d} \phi_{x}(1)\left[\tilde{b}\left(\alpha_{0}\right)-b_{0}\right]}{\lambda \bar{\ell}^{3}+V_{d} \bar{\ell}^{2}}+\eta_{0}\left(\gamma_{b}+\lambda\right) \int_{0}^{1} \phi .
$$

Replacing $\phi_{x}(0)$ by $\phi_{x}(1)+\bar{\ell} E_{2} \phi$ above and using the fact that $\phi_{x}(1) \geq 0$, we have

$$
\begin{aligned}
0 & <-\frac{\left[b_{0} V_{d}+\lambda \bar{\ell} \tilde{b}\left(\alpha_{0}\right)\right]\left[\phi_{x}(1)+\bar{\ell} E_{2} \phi\right]}{\lambda \bar{\ell}^{3}+V_{d} \bar{\ell}^{2}}-\frac{V_{d} \phi_{x}(1)\left[\tilde{b}\left(\alpha_{0}\right)-b_{0}\right]}{\lambda \bar{\ell}^{3}+V_{d} \bar{\ell}^{2}}+\eta_{0}\left(\gamma_{b}+\lambda\right) \int_{0}^{1} \phi \\
& =-\left[\frac{V_{d} b_{0}+\lambda \bar{\ell} \tilde{b}\left(\alpha_{0}\right)}{\lambda \bar{\ell}^{2}+V_{d} \bar{\ell}}\right] E_{2} \phi-\frac{\left(V_{d}+\lambda \bar{\ell}\right)}{\lambda \bar{\ell}^{3}+V_{d} \bar{\ell}^{2}} \phi_{x}(1) \tilde{b}\left(\alpha_{0}\right)+\eta_{0}\left(\gamma_{b}+\lambda\right) \int_{0}^{1} \phi \\
& \leq\left\{\left[\frac{V_{d} b_{0}+\lambda \bar{\ell} \tilde{b}\left(\alpha_{0}\right)}{\lambda \bar{\ell}^{2}+V_{d} \bar{\ell}}\right] \omega+\eta_{0}\right\}\left(\gamma_{b}+\lambda\right) \int_{0}^{1} \phi \\
& =\left\{\frac{V_{d} b_{0}+\lambda \bar{\ell} \tilde{b}\left(\alpha_{0}\right)}{b_{0} V_{d}+\left(\lambda+2 \gamma_{b}\right) \bar{\ell} \int_{0}^{1} \tilde{b}}+\frac{\left(\lambda+2 \gamma_{b}\right) \bar{\ell} \int_{0}^{\alpha_{0}} \tilde{b}-\lambda \bar{\ell} \alpha_{0} \tilde{b}\left(\alpha_{0}\right)}{b_{0} V_{d}+\left(\lambda+2 \gamma_{b}\right) \bar{\ell} \int_{0}^{1} \tilde{b}}\right\}\left(\gamma_{b}+\lambda\right) \int_{0}^{1} \phi .
\end{aligned}
$$


Since $V_{d} b_{0}+\lambda \bar{\ell} \tilde{b}\left(\alpha_{0}\right) \geq 0$, therefore if $\lambda \leq 0$, the terms inside the curly bracket are positive. If $\lambda>0$, then since $\alpha_{0}<1$, the terms inside the curly bracket are also positive. Therefore, $\int_{0}^{1} \phi>0$ so that $E_{2} \phi<0$. This contradicts the subcase we are in. The proof of Lemma 3.2 is complete.

\section{Non-existence of COMPlex Eigenvalues}

The eigenvalue problem (2.7) is non-local and non-selfadjoint. Although we are unable to show that all its eigenvalues are real, we will, in this section, present strong numerical evidence that this is indeed the case.

We divide $[0,1]$ into 1000 equal parts, discretize (2.7) and use Matlab to find the eigenvalues of the resulting matrix. We did this for $b_{0}$ in a wide range of values, and all 1000 eigenvalues were found to be real and negative. We also obtained asymptotic formulas for the eigenvalues as $b_{0} \rightarrow \infty$ and compared the results obtained from using these formulas with numerics in Table 1.

TABLE 1. Comparison of 10 largest eigenvalues for $b_{0}=500$

\begin{tabular}{|r|r|r|}
\hline Using formal asymptotic & Solving (2.7) numerically & Relative Error in \% \\
\hline-27986.970799 & -27948.659114 & 0.136891 \\
\hline-22113.162113 & -22082.983561 & 0.136473 \\
\hline-16930.389743 & -16907.269524 & 0.136560 \\
\hline-12438.653688 & -12421.525704 & 0.137700 \\
\hline-8637.953950 & -8625.854456 & 0.140074 \\
\hline-5528.290528 & -5520.212489 & 0.146122 \\
\hline-3109.663422 & -3104.763214 & 0.157580 \\
\hline-1382.072632 & -1379.315927 & 0.199462 \\
\hline-345.518158 & -344.655110 & 0.249784 \\
\hline-1.800790 & -1.803155 & 0.131375 \\
\hline
\end{tabular}

In the next table, we document the eigenvalues when $b_{0}$ is small. Since the traveling cell solution develops a boundary layer at $y=0$ as $b_{0} \rightarrow 0$, we refine our mesh points to ensure that our numerical results are trustworthy.

TABLE 2. Comparison of 10 largest eigenvalues for $b_{0}=1$

\begin{tabular}{|r|r|r|}
\hline Using 2000 mesh points & Using 3000 mesh points & Relative Error in \% \\
\hline-64.26655 & -64.13063 & 0.212 \\
\hline-54.20776 & -54.15408 & 0.099 \\
\hline-42.88961 & -42.79822 & 0.214 \\
\hline-35.90944 & -35.92078 & 0.032 \\
\hline-26.76623 & -26.69850 & 0.254 \\
\hline-22.98394 & -23.04107 & 0.248 \\
\hline-16.25227 & -16.18816 & 0.396 \\
\hline-14.97859 & -15.07460 & 0.637 \\
\hline-11.98912 & -12.01627 & 0.226 \\
\hline-0.18120 & -0.18239 & 0.652 \\
\hline & & \\
\hline
\end{tabular}

In conclusion, we believe that all the eigenvalues of (2.7) are real. 


\section{REFERENCES}

[1] Choi,Y.S., Juliet Lee and Roger Lui (2004). Traveling wave solutions for a one-dimensional crawling nematode sperm cell model. J. Math. Bio. 49, 310-328. MR2102761 (2005g:92011)

[2] Choi, Y.S., Patrick Groulx and Roger Lui (2005). Moving boundary problem for a onedimensional crawling nematode sperm cell model. Nonlinear Analysis: Real World Applications 6, 874-898. MR2165218 (2006f:35283)

[3] Mogilner, A. and D. W. Verzi (2003). A Simple 1-D Physical Model for the Crawling Nematode Sperm Cell. J. Stat. Phys. 110, 1169-1189.

Department of Mathematics, University of Connecticut, Storrs, Connecticut 06269

E-mail address: choi@math.uconn.edu

Department of Mathematical Sciences, Worcester Polytechnic Institute, WorcesTer, Massachusetts 01609

E-mail address: rlui@wpi.edu 